\title{
Unilateral thalamic glioma disrupts large-scale functional architecture of human brain during resting state
}

This article was published in the following Dove Medical Press journal: Neuropsychiatric Disease and Treatment

Sirui Li

Lei Gao

Ying Liu

Yawen Ao

Haibo Xu

Department of Radiology, Zhongnan Hospital of Wuhan University, Wuhan University, Wuhan 43007I, People's

Republic of China
Correspondence: Haibo Xu Department of Radiology, Zhongnan Hospital of Wuhan University, Wuhan University, Wuhan 43007I, People's Republic of China

Email xuhaibol I20@hotmail.com
Background: The thalamus is an important deep brain structure for the synchronization of brain rhythm and the integration of cortical activity. Human brain imaging and computational modeling have non-invasively revealed its role in maintaining the cortical network architecture and functional hierarchy.

Purpose: The objective of this study was to identify the effect of unilateral thalamic damage on the human brain intrinsic functional architecture.

Patients and methods: We collected an 8-minute resting-state functional magnetic resonance imaging (R-fMRI) data on a 3.0 T magnetic resonance scanner for all the participants: a preoperative patient with left thalamus destroyed by anaplastic astrocytoma (WHO grade III type of astrocytoma) and 20 matched healthy controls. The R-fMRI data was analyzed for functional connectivity and amplitude of spontaneous fluctuations.

Results: The patient showed prominent decrease in functional connectivity within primary sensory networks and advanced cognitive networks, and extensive alterations in between-network coupling. Further analysis of the amplitude of spontaneous activity suggested significant decrease especially in the topographies of default mode network and the Papez circuit.

Conclusion: This result provided evidence about the consequences of thalamic destruction on the correlation and landscape of spontaneous brain activity, promoting our understanding of the effects of thalamic damage on large-scale brain networks.

Keywords: brain networks, functional connectivity, default mode network, Papez circuit

\section{Introduction}

Over the past decade, investigation of the human brain functional architecture and network neuroscience has been a major goal of neuroscience research. ${ }^{1-4}$ Support for this connectional perspective on brain function comes from studies of resting-state functional magnetic resonance imaging (R-fMRI), or intrinsic fluctuations of blood oxygen level dependent (BOLD) signal..$^{5}$ Insights from the R-fMRI studies have brought out some functional attributes of human brain function, including large-scale intrinsic networks, and the temporal-spatial structure of the intrinsic BOLD signals. ${ }^{1-4}$

The brain's repertoire of intrinsic functional architecture is ultimately determined by its structural underpinnings. ${ }^{6,7}$ Structural damage (such as those caused by stroke and tumor) often leads to varying degrees of brain dysfunction, depending on the change in topography and extent of the injury. Evidence based on computational neuroscience suggests that damage at the core/central nodes of human brain network, such as the anterior cingulate cortex, precuneus/posterior cingulate cortex, anterior insula, and the thalamus, often leads to collapse of the network. ${ }^{2,8-10}$ 
Out of all of these important core/central regions, the impact of thalamic damage on the functional architecture of the human brain is yet poorly understood. The thalamus is a primary relay station between the widespread cerebral cortex and subcortical regions, projects to almost all cortical and neocortical regions, maintains neuronal synchronization and well-organized functional networks, and integrates a wide range of cortical information to regulate consciousness, sleep, and wakefulness. ${ }^{8-11}$ Zhang et al ${ }^{12}$ first mapped the intrinsic functional connectivity projection of specific thalamus subregions and the cerebral cortex, as well as developmental and maturation trajectories of the thalamocortical functional connectivity. ${ }^{13}$ One previous report focused its attention on the resting connectivity in a single multiple sclerosis patient with an anterior thalamic lesion, in particular in the default mode network (DMN). ${ }^{14}$ Unfortunately, despite the central role of the thalamus, information on the effect of the entire thalamic lesion on the brain's intrinsic activity is still extremely lacking. Thus, an important question arises: how does the destruction of the unilateral thalamus affect the functional operations of the human brain?

We hypothesized that thalamic destruction specifically results in dysfunction of the large-scale network and oscillations of intrinsic brain activity measured by R-fMRI. To explore this issue, we used a unique clinical case, a male patient with left thalamus destroyed by anaplastic astrocytoma (WHO grade III type of astrocytoma).

\section{Methods}

\section{Ethics statement}

The present study was approved by the Medical Ethics Committee of Zhongnan Hospital of Wuhan University, and written informed consent in accordance with the 1975 Declaration of Helsinki (and as revised in 1983) following a complete description of the measurement was obtained from all participants. The patient signed a written informed consent form that included permission to publish the case details and any accompanying images.

\section{Subjects}

A 30-year-old man was admitted to the hospital with a problem of headache and sleepiness since half a month. The patient complained of decreased vision and tinnitus, with decreased memory and distracted attention. He had no history of central nervous system infection, systemic disease, or congenital/chromosomal abnormality. Structural MRI demonstrated left thalamic space-occupying lesions, with long T1 and long T2 signals and a higher T2 fluid-attenuated inversion recovery (FLAIR) signal. A postcontrast T1-weighted scan revealed intra-focal partial/focal annular enhancement, while the rest did not enhance. The imaging characteristics indicated the diagnosis of glioma. Subsequently, the patient underwent thalamic tumor resection, and the postoperative pathology confirmed anaplastic astrocytoma (WHO grade III) (Figure 1). To establish differences between the individual case and its matched healthy controls, similar to the confidence interval reference range for establishing healthy controls, we carefully selected 20 healthy male controls (age range $=19-45$ years; mean age $=31.2$ years) with matched age and education. All subjects had normal hearing and vision and were right-handed as determined by the Edinburgh Handedness Inventory test. ${ }^{15}$

\section{Functional MRI data acquisition}

In all participants, T1 high-resolution anatomical and R-fMRI images were acquired on a Siemens Trio 3.0 T magnetic resonance scanner (Siemens, Munich, Germany). Head movement was restricted using a pillow and foam, and earplugs were used to attenuate scanner noise and maximize patient comfort. During the resting-state functional scans, all subjects were asked to close their eyes, stay awake, and remain as still as possible. A total of 8 minutes of T2*-weighted BOLD fMRI images were obtained (33 axial slices, 3.8-mm slice thickness with a 0.3 -mm gap, 2-s recovery time (TR), 240 volumes). A T1 magnetization prepared rapid gradient echo sequence was also acquired in the same session for co-registration with functional data (176 sagittal slices, 1-mm in-plane resolution).

\section{Preprocessing of $\mathrm{M} M \mathrm{RI}$ data}

R-fMRI data were processed using DPABI (rfmri.org/ dpabi), ${ }^{16}$ Statistical Parametric Mapping (SPM12, http:// www.fil.ion.ucl.ac.uk), and MATLAB (https://www. mathworks.com), and followed conventional methods as previously described. ${ }^{17}$ The methods mainly included the following steps: 1) discarding the first $10 \mathrm{TRs}$; 2) correcting for slice acquisition shifts; 3 ) realigning and correcting for small movements between scans, subjects with a maximum displacement in the $\mathrm{x}, \mathrm{y}$, or $\mathrm{z}$ direction of more than $2 \mathrm{~mm}$ or more than $2^{\circ}$ of angular rotation about any axis for any of the 230 volumes were excluded from this study (no subject was excluded according to this criterion); 4) co-registering for individual $\mathrm{T} 1$ and the realigned functional volumes; 5) normalizing to the Montreal Neurological Institute (MNI152) space through Diffeomorphic Anatomical Registration Through Exponentiated Lie Algebra (DARTEL), ${ }^{18}$ 6) spatial smoothing (6 mm full-width half-maximum 


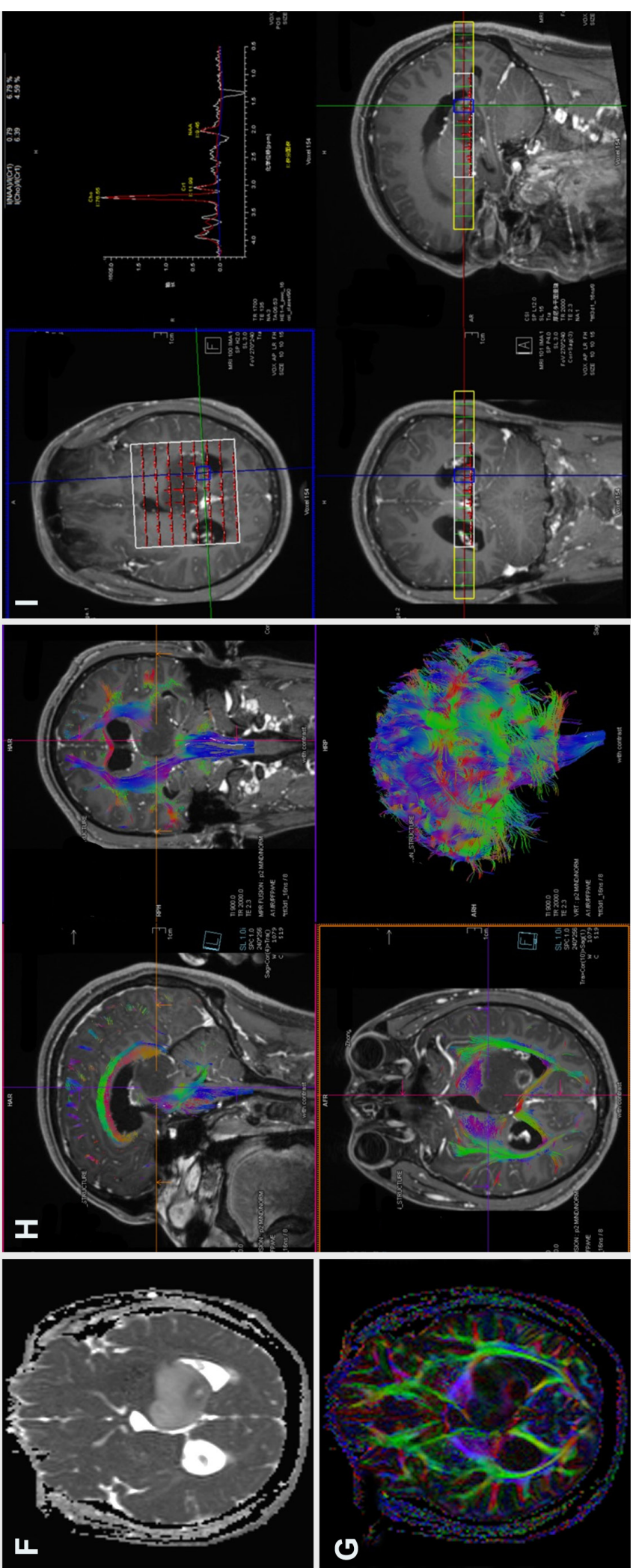

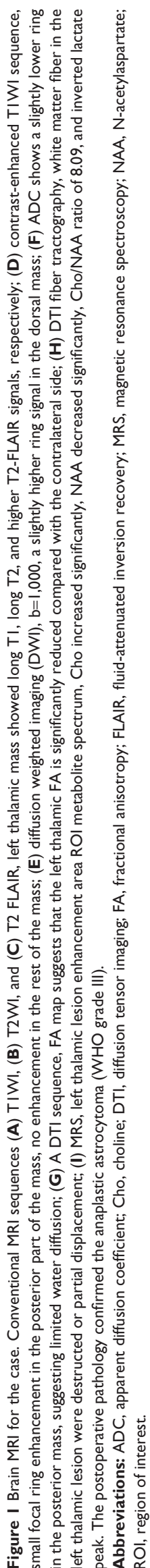


Gaussian blur in each direction); 7) reducing confounding factors via linear regression, including the signals from the white matter and cerebrospinal fluid and linear and quadratic trends; 8$)$ temporal filtering $(0.01-0.1 \mathrm{~Hz})$ of the time series; and 9) motion scrubbing with a frame-wise displacement (FD) threshold of 0.5 . The clean and low-frequency filtered resting functional volumes were used for further calculations. It should be noted that, for the patient, we made a left thalamic mask to avoid the influence of the lesion on the functional-structural registration.

\section{Seed-based analysis}

We began our analysis by computing seed-based functional connectivity. Based on our hypothesis and the patient's syndrome, we placed a set of seed regions on the healthy side (right hemisphere), including the default, dorsal attention, visual, auditory, salience, and the language systems, as previously described. ${ }^{19,20}$ The seeds were 6-mm-diameter spheres centered on previously published foci (Table 1). ${ }^{19,20}$ Functional connectivity maps were produced by extracting the BOLD time course from a seed region, and then computing the correlation coefficient between that time course and the time course from all other brain voxels.

\section{ROI-ROI correlation analysis}

To characterize the integrity and alterations of the largescale functional brain architecture, we analyzed the region of interest (ROI)-wise functional connectivity. We selected twenty 6-mm spherical seed regions with the center coordinates on the dorsal attention, default, somatomotor, visual, auditory, and executive control networks (Table 2). ${ }^{19}$ The BOLD time series were extracted from each seed region, and then the correlation coefficients between the ROI pairs were calculated. The obtained correlation matrix which represented large-scale brain connectivity was then transformed using Fisher's z-shift for further individual versus group statistics.

Table I Seed regions and coordinates for seed-based analysis

\begin{tabular}{|c|c|c|}
\hline System & Seed & $\begin{array}{l}\text { Talairach } \\
\text { coordinates }(\mathbf{R})\end{array}$ \\
\hline Somatomotor & Right somatomotor & $(39,-26,51)$ \\
\hline Default & $\begin{array}{l}\text { Right posterior } \\
\text { cingulate/precuneus }\end{array}$ & $(4,-40,43)$ \\
\hline Language & Right inferior frontal gyrus & $(48,-|3,3|)$ \\
\hline Visual & Right VI & $(20,75,12)$ \\
\hline Auditory & Right Al & $(50,-25,8)$ \\
\hline Dorsal attention & Right intraparietal sulcus & $(21,-69,30)$ \\
\hline
\end{tabular}

Notes: System, seed name, and Talairach coordinates for seed regions used in this analysis are shown. These seed regions and coordinates were from Pizoli et al. ${ }^{20}$ Abbreviation: R, right.
Table 2 Seed regions and coordinates for ROI-wise analysis

\begin{tabular}{|c|c|c|c|}
\hline System & Seed & BA & $\begin{array}{l}\text { Talairach coordinates (L) } \\
\text { (R) }\end{array}$ \\
\hline \multirow[t]{3}{*}{ Dorsal attention } & FEF & 6 & $(-24,-12,57)(28,-7,54)$ \\
\hline & IPS & 7 & $(-23,-66,46)(25,-58,52)$ \\
\hline & $M T+$ & $19 / 37$ & $(-45,-69,-2)(45,-69,-4)$ \\
\hline \multirow[t]{3}{*}{ Default } & MPF & $32 / 10$ & $(-3,39,-2)(I, 54,21)$ \\
\hline & LP & 39 & $(-47,-67,36)(53,-67,36)$ \\
\hline & PCC & 31 & $(-5,-49,40)$ \\
\hline Somatomotor & SM & $4 / 3,1,2$ & $(-39,-26,5 \mathrm{I})(38,-26,48)$ \\
\hline Visual & VI & 17 & $(-19.5,-75,12)(16.5,-72,12)$ \\
\hline Auditory & Al & 41 & $(-50,-25,8)(50,-25,8)$ \\
\hline \multirow[t]{2}{*}{ Executive control } & $\mathrm{dACC}$ & 32 & $(-1,10,46)$ \\
\hline & OP & & $(-35,14,5)(36,16,4)$ \\
\hline
\end{tabular}

Notes: System, seed name, BA, and Talairach coordinates for seed regions used in this analysis are shown. These seed regions and coordinates were from Johnston et al. ${ }^{19}$

Abbreviations: BA, Brodmann's area; L, left; R, right; ROI, region of interest.

\section{Amplitude of low-frequency fluctuations analysis}

Finally, to analyze the effect of unilateral thalamic lesion on the local and whole brain spontaneous BOLD activity, we calculated the amplitude of the low frequency fluctuations (ALFF). ${ }^{21}$ ALFF mainly characterize the power of the low-frequency BOLD signal, which is supposed to indirectly reflect the extent or intensity of local intrinsic brain activity. ${ }^{16,21}$

\section{Statistical analysis}

Since this study was for a single case analysis, we used an unpaired $t$-test with equal variance to examine the extent to which individual voxel and connectivity were deviated from its matched healthy controls. We defined the $95 \%$ confidence interval based on individual voxel and connectivity measures derived from healthy controls.

\section{Results}

\section{Seed-based functional connectivity}

Figure 2 depicts seed-based analysis, the seed regions settings, and seed-based functional connectivity profiles for the patient and healthy controls. The average correlation map of the seed-based functional connectivity of the healthy controls presents a characteristic layout consistent with previous reports. It can be found that the functional connectivity map of almost all six seed regions (right primary somatomotor, auditory, visual, intraparietal sulcus, inferior frontal gyrus, and posterior cingulate cortex) of the patient shows a decrease in functional connectivity, especially more prominent on the ipsilateral side of the patient's thalamic injury. 


\section{Seed-based functional connectivity}

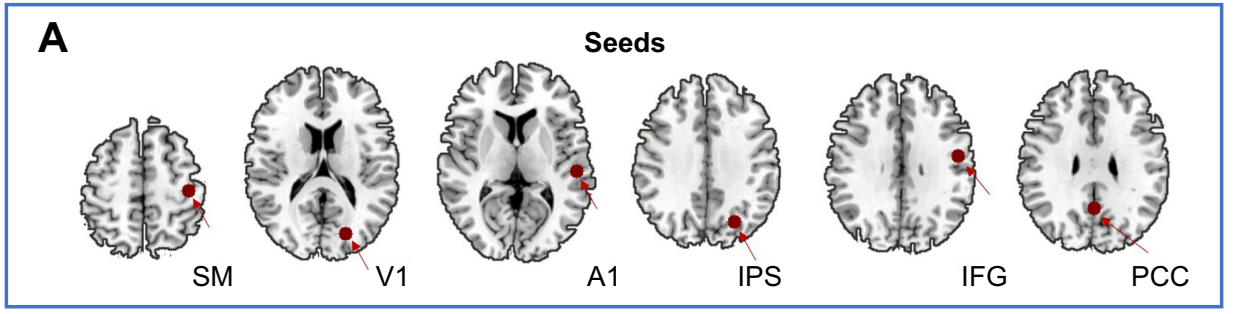

B



C

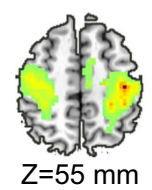

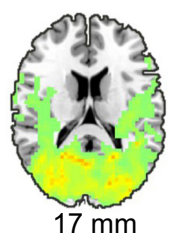

Controls
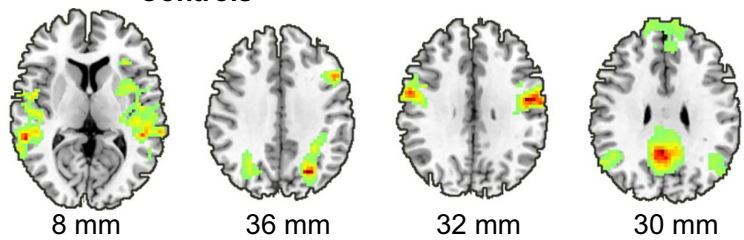

Figure 2 Seed-based functional connectivity. (A) Schematic of seed region selection, these 6-mm-diameter spherical regions include the right primary SM, AI, VI, IPS, IFG, and PCC, whose functional roles are involved in the somatomotor, auditory, visual, dorsal attention, language, and default systems, respectively. (B) The topographies where time courses in the patient's seed region is positively correlated $(r=0.5)$ with other voxels in the whole brain, which are large-scale brain network systems including the somatomotor, auditory, visual, dorsal attention, language, and default systems; visually, these networks show prominent reductions in the contralateral functional connectivity corresponding to the seed regions (ie, ipsilateral functional connectivity). (C) Group average functional connectivity maps (correlation maps) of the healthy controls, the topographies where time courses in the seed region is positively correlated $(r=0.5)$ with other voxels in the whole brain, outlines typical large-scale brain networks, as previous described and reported.

Abbreviations: AI, auditory; IFG, inferior frontal gyrus; IPS, intraparietal sulcus; PCC, posterior cingulate cortex; SM, somatomotor; VI, visual.

\section{Pair wise ROI-ROI correlations}

The patient and healthy controls' ROI-wise functional connectivity matrix and topographies are shown in Figure 3. Evidently, the controls showed typical connectional patterns of resting-state fluctuations within and between functional brain systems as previously reported. In contrast, the patient presented a less well-organized network architecture (Figure 3, right panel). Specifically, the connectivity matrix of the patient exhibited remarkably decreased functional connectivity within the default, salience, dorsal attention, and visual networks, and decreased functional connectivity between the salience and dorsal attention networks, as well as increased functional connectivity between default mode and salience networks.

\section{Amplitude of low-frequency fluctuations analysis}

Remarkable alterations in functional connectivity are seen, considering the nature of the thalamus in regulating cortical rhythm. We raised the question of whether the amplitude of the low frequency BOLD signal contributes to their changes. To explore this issue, we then compared the difference between patient and controls in both voxel- and ROI-levels of ALFF. Gray matter-based voxel-level analysis showed significantly reduced ALFF in the patient in bilateral posterior cingulate gyri, bilateral hippocampus, anterior cingulate gyrus, bilateral lateral parietal, and temporalparietal conjunctions (Figure 4). It is worth noting that these topographies largely overlapped with the default network (medial prefrontal cortex precuneus/posterior cingulate cortex, and bilateral lateral parietal cortices) and the so-called Papez circuit (hippocampal formation, fornix, mammillary bodies, mammillothalamic tract, anterior thalamic nucleus, cingulum, entorhinal cortex, hippocampal formation). The average ALFF values extracted from 46 classic cortical ROIs also suggest a significantly uniform ALFF reduction within the default network, and isolated nodes from other systems (Figure 5).

\section{Discussion}

Non-invasive MR human brain imaging brings an unprecedented opportunity to understand the human brain architecture. 


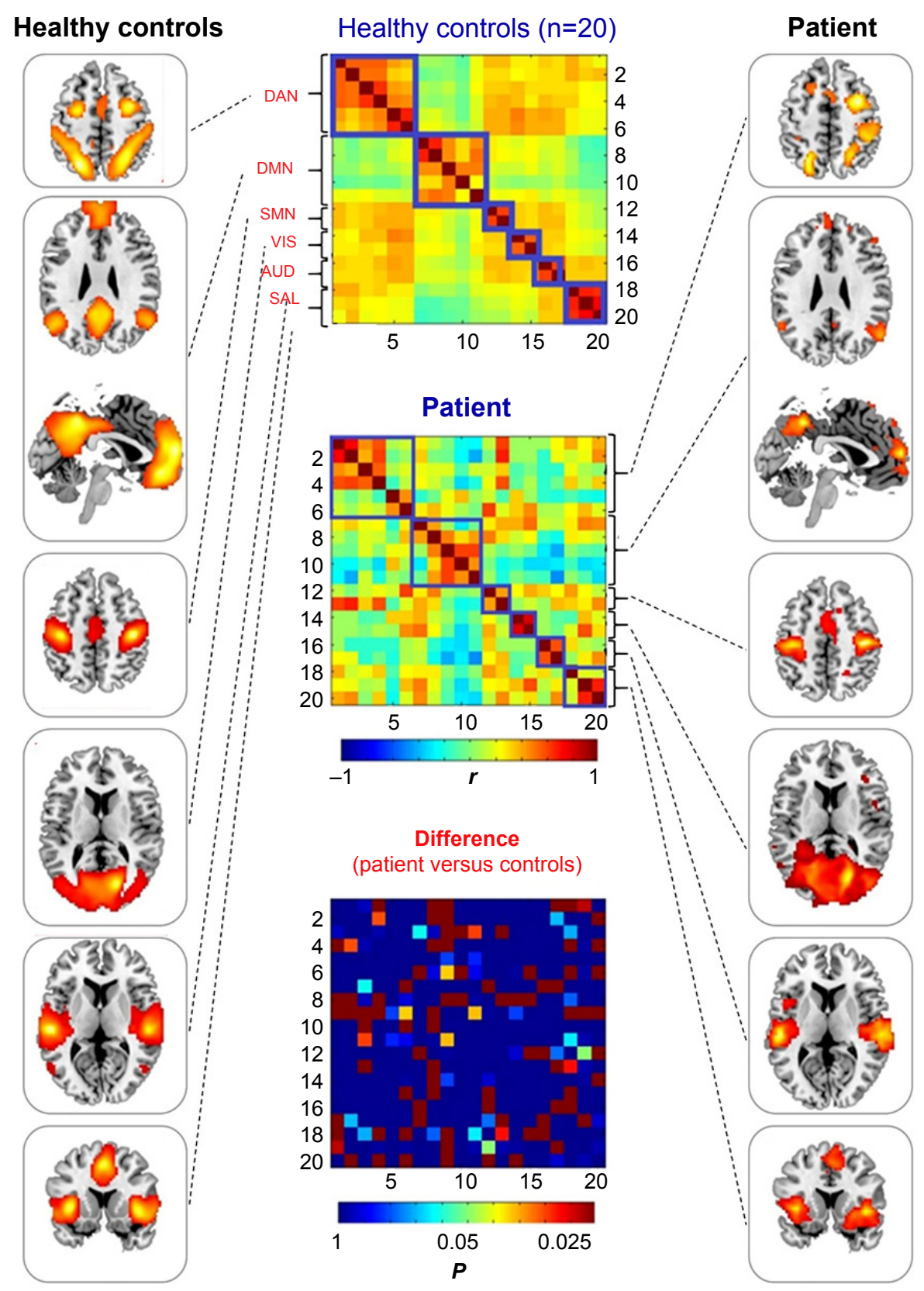

Figure 3 ROI-based large-scale brain networks. Left panel: group average of ROI-based functional connectivity in healthy controls, topographies of large-scale brain networks including dorsal attention, default, somatomotor, visual, auditory, and salience systems from top to bottom. Middle panel: functional connectivity matrices ( 20 nodes $\times 20$ nodes correlation) of healthy controls' mean (top), patient (middle), and between-group difference in patient versus controls comparison via Z-transformation (bottom, the elements in this matrix have been transformed into corrected $P$-values, with only $P \leq 0.05$ coloring, and $P>0.05$ colored with uniform dark blue). Right panel: ROI-based functional connectivity in the patient, topographies of large-scale brain networks including dorsal attention, default, somatomotor, visual, auditory, and salience systems from top to bottom.

Abbreviations: AUD, auditory network; DAN, dorsal attention network; DMN, default mode network; ROI, region of interest; SAL, salience network; SMN, somatomotor network; VIS, visual network.

One of the important research domains is the relationship between human brain structure and function. Empirical data and computational neuroscience have revealed that, in complex human brain networks, some hub nodes are critical for maintaining the integrity and dynamics of the functional architecture. In this study, we presented a case to investigate how the damage of the unilateral thalamus would affect the large-scale resting of the human brain.
Using an 8-minute scan of resting-state fMRI data, we analyzed the spontaneous brain activity architecture from three levels: 1) seed-based univariate analysis of a given network within and between hemispheric functional connectivity, 2) large-scale cortical network based on ROI pairs correlations, and 3) amplitude and distribution of these spontaneous neural activities. Consistent with our hypothesis, unilateral thalamic injury significantly reduced 


\section{Amplitude of low-frequency fluctuations analysis}

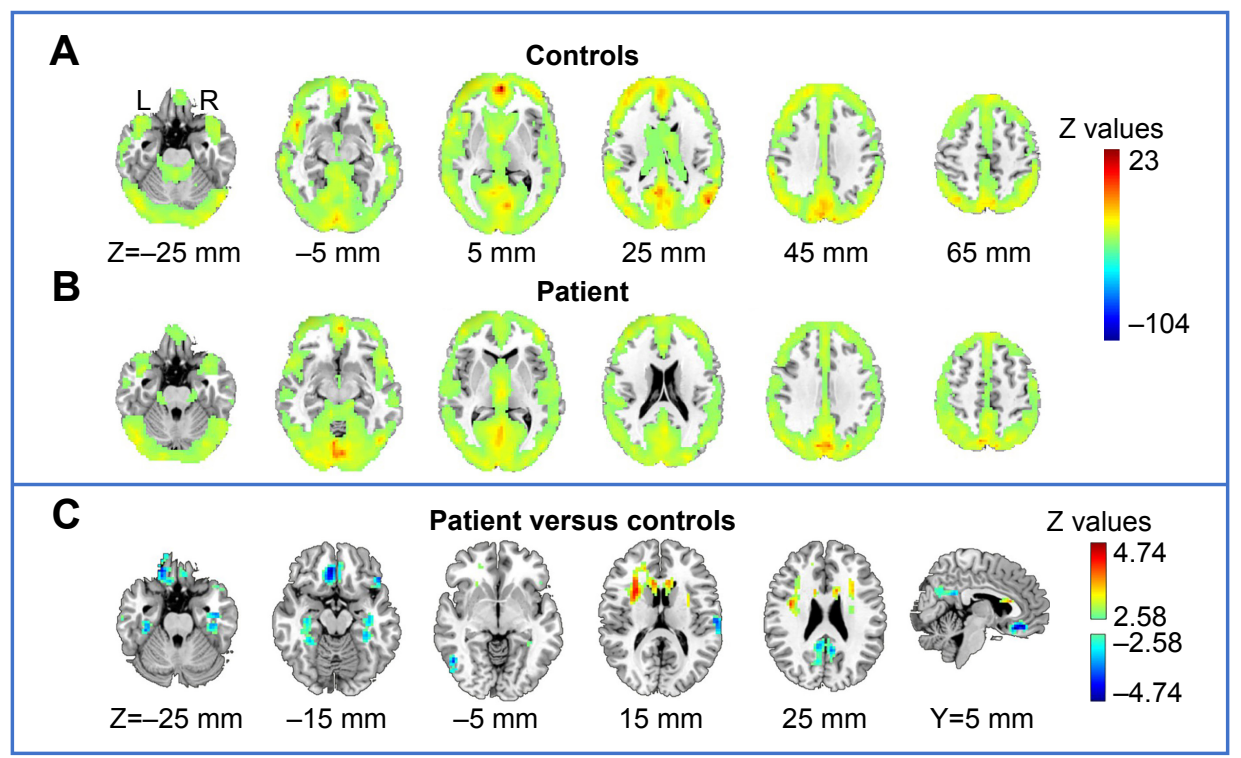

Figure 4 Amplitude of low-frequency fluctuations analysis. (A) Average gray matter resting fluctuations amplitude in the healthy controls. (B) Gray matter resting fluctuations amplitude in the patient. (C) Between-group comparison of patient versus controls via Z-transformation.

Note: $Y$ and $Z$ represent sagittal and axial coordinates, respectively, in standard brain template space.

Abbreviations: L, left; $R$, right.

functional connectivity in the sensory (visual, auditory, and somatomotor) and cognitive networks (dorsal attention, default, and salience), meanwhile suppressed cortical spontaneous BOLD fluctuations were also found in this patient.

\section{Effects of thalamic lesions on resting-state functional connectivity}

Thalamic injury significantly disrupts the large-scale neuronal networks (the canonical signature of low-frequency

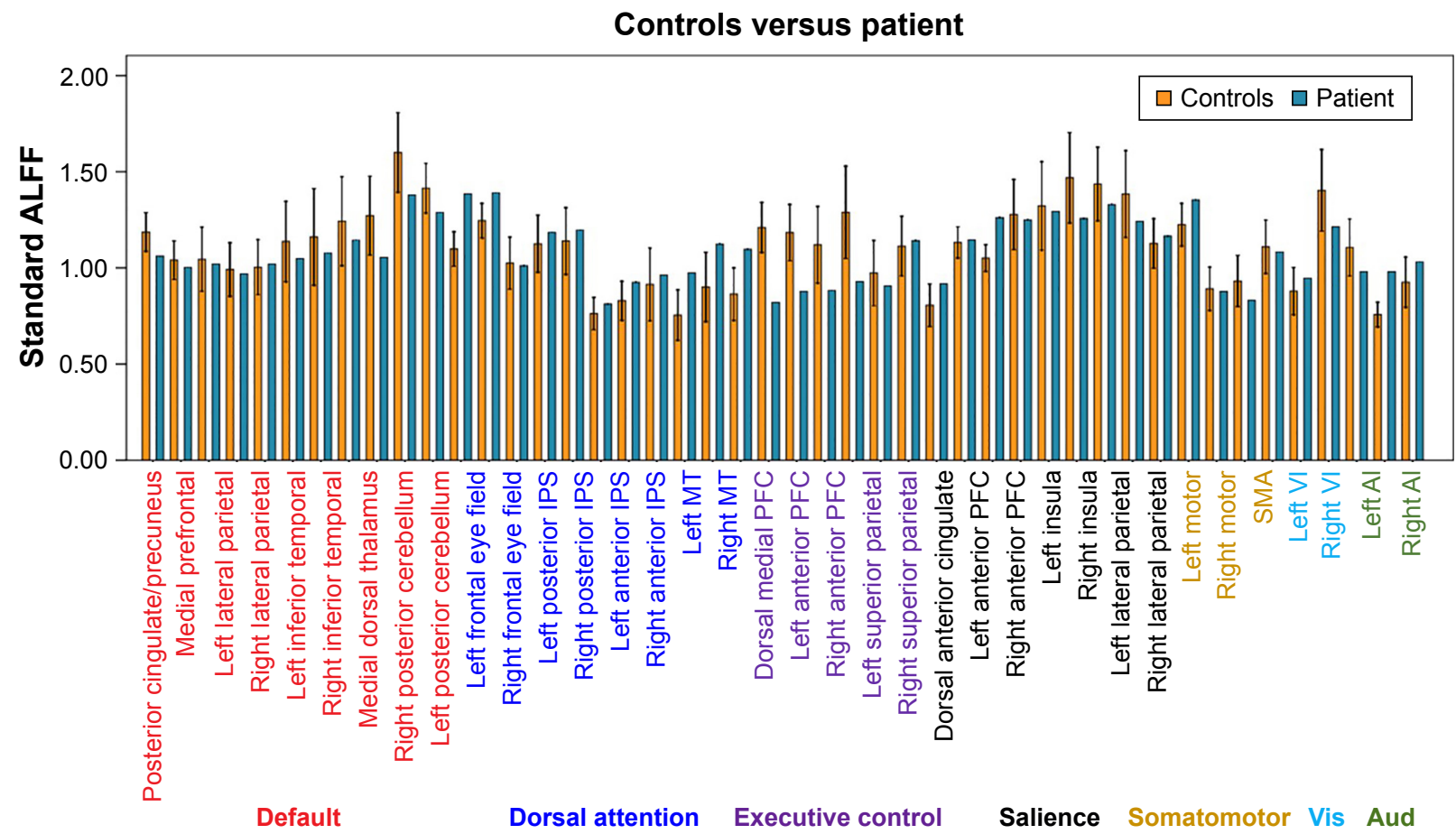

Figure 5 ALFF signals over predefined ROls. The average values in the patient (blue) and controls (orange) across 46 6-mm-diameter spherical canonical ROls (all in gray matter), ${ }^{20}$ corresponding to previously described nodes within the default, dorsal attention, executive control, salience, somatomotor, primary visual, as well as primary auditory networks (labeled in the bottom panel). The error bars indicate $95 \%$ confidence interval for the ALFF values derived from the healthy controls.

Abbreviations: AI, auditory; ALFF, amplitude of the low frequency fluctuations; IPS, intraparietal sulcus; MT, middle temporal region; PFC, prefrontal cortex; ROI, region of interest; VI, visual. 
fluctuations patterns), which mostly reduce within and between the brain network systems. One previous study has also focused its attention on resting connectivity between a single multiple sclerosis patient with an anterior thalamic lesion and a group of controls, in particular, in the default mode network. ${ }^{14}$ In this particular case of our study, we presented a complete glioma-induced left thalamic destruction that extended the range of damage, and thus the damage spectrum spanned the default network and involved multiple large-scale brain networks of both advanced cognitive functional networks and primary sensory networks. Widespread reduction in cortical functional connectivity is thought to be responsible for communication and goal-directed behavioral disorders, including physical disconnection caused by traumatic brain injury, ${ }^{22}$ reduced occipital inputs due to early blindness, ${ }^{23}$ reduced sensory motor functionality in early stage limb-onset amyotrophic lateral sclerosis, ${ }^{24}$ and the multidimensional sensory abnormalities caused by acute infarction or bleeding of the unilateral posterolateral thalamus. ${ }^{14}$

The thalamus is an important node on the basal ganglia circuitry, ${ }^{8}$ it not only promotes the transmission of almost all cortical inputs, including primary sensory, and advanced cognitive functions but also facilitates the regulation and integration of this information. Cortical-thalamic-cortical loops are another important way for the thalamus to function, promoting sensorimotor and advanced cognitive input information to the neocortex. ${ }^{9,11,25}$ Previous functional ${ }^{12}$ and diffusion $^{26}$ MRI have established functional connectivity and fiber tracking projections of the thalamus-cortex. Based on these findings, prominent changes were reported in various physiological states, ${ }^{27}$ development, ${ }^{13,28,29}$ and brain diseases. ${ }^{30-32}$

\section{Altered resting spontaneous activity}

The widespread reduction in cortical functional connectivity raised a question whether that may reflect changes in the information propagation by the thalamic gating, and we continued to investigate the amplitude of this spontaneous neural activity (ie, the ALFF). We found that the overall ALFF in the patient's gray matter ROIs was generally lower than the mean of the healthy controls +2 standard deviations. Further voxel-based statistical analysis found that the topographies of the patient with significantly reduced ALFF included posterior cingulate gyrus, bilateral hippocampus, anterior cingulate gyrus, bilateral lateral parietal and temporal-parietal conjunctions. It is worth noting that these topographies largely overlapped with the default network and the so-called Papez circuit.
On the other hand, results of the ALFF analysis are from those of functional connectivity, whose findings involved multiple large-scale brain networks. One main reason may be that the unilateral thalamic lesion disrupts the subcortical information outputs and cortical communication and integration, leading to frequency changes in neuronal assembly oscillations.

The default brain network was traditionally found to be a negative activation while performing goal-directed tasks, possibly as a baseline activity at rest. ${ }^{33}$ Later clinical applications in the neuroimaging community have found characteristic changes (mainly reduced) of the default network in memory impairment-associated conditions including Alzheimer's disease, altered coupling of default network, and other large-scale networks in attention deficit hyperactivity disorder and autism (for a systemic review, please see Raichle $^{3}$ ). Especially in the past decade, graph theory analysis (based on human brain connectome data) and cluster analysis have identified that DMN is at the core of the human brain functional hierarchy. ${ }^{2}$ Recent studies have recognized lag structure in the human intrinsic architecture, the DMN is supposed to be an early spatiotemporal source, promoting the propagation of neural information to other large-scale cortical networks (later spatiotemporal sources). ${ }^{4,34}$ A number of previous studies have reported focally reduced ALFF in various neuropsychiatric conditions. ${ }^{35,36}$ It is highly likely that these effects are related to the decreased local activity and shifted frequency band..$^{37,38}$ In summary, ALFF anomalies mainly occur in the DMN and the Papez circuit as these regions may serve as a source of spontaneous signaling, mediating the integration of other sensory and cognitive networks, cortical rhythms of inhibitory control, and coordination.

This report has important clinical implications, as previous studies have focused on intact brain and animal experiments in the thalamus. This study provides insight into understanding the effects of unilateral thalamic destruction on the amplitude and correlation patterns (connectivity) of resting spontaneous neural activity. Thalamic stimulation therapy for disorders, Parkinson's disease, etc. provides theoretical evidence, and also provides a potentially useful tool for understanding its targeted stimulation.

\section{Conclusion}

Unilateral thalamic damage suppresses the amplitude of spontaneous neuronal activity, especially in the default network and Papez circuit, as well as the integrity and coupling of large-scale functional networks, and functional connectivity within the ipsilateral side of injury. This result provided evidence about the consequences of thalamic destruction on 
the correlation and landscape of spontaneous brain activity, thus promoting our understanding of the effects of thalamic damage on large-scale brain networks.

\section{Acknowledgments}

This study has been supported by the National Natural Science Foundation of China (under Grant Nos 81771819 and 81571734), National key research and development plan of China (Project 2017YFC0108803), Zhongnan Hospital of Wuhan University Science, Technology and Innovation Seed Fund (Projects cxpy2017048 and cxpy20160057), and the Fundamental Research Funds for the Central Universities (Projects 2042017kf0284 and 2016060605100525). We thank all members of the Departments of Radiology and Neurosurgery at Zhongnan Hospital of Wuhan University for conducting this study as well as all participants for their good collaboration.

\section{Author contributions}

SL, LG, and YL planned and conducted statistical analyses, wrote the draft of the manuscript including creation of the figures, and modified all subsequent drafts. LG processed all MRI data. HX designed the study. YL and YA reviewed the final draft of the manuscript. SL and YA collected the MRI data. HX supervised data analyses, substantially contributed to interpretation of the results, and made substantial modifications to all drafts of the manuscript. All authors contributed to data analysis, drafting and revising the article, gave final approval of the version to be published, and agree to be accountable for all aspects of the work.

\section{Disclosure}

The authors declare no potential conflicts of interest with respect to the research, authorship, and/or publication of this article. The authors report no other conflict of interest in this work.

\section{References}

1. Mitra A, Raichle ME. How networks communicate: propagation patterns in spontaneous brain activity. Philos Trans $R$ Soc Lond B Biol Sci. 2016;371(1705):20150546. doi:10.1098/rstb.2015.0546

2. Bassett DS, Sporns O. Network neuroscience. Nat Neurosci. 2017;20(3): 353. doi: $10.1038 / \mathrm{nn} .4465$

3. Raichle ME. The brain's default mode network. Annu Rev Neurosci. 2015;38:433-447. doi:10.1146/annurev-neuro-071013-014030

4. Raichle ME. The restless brain: how intrinsic activity organizes brain function. Philos Trans R Soc Lond B Biol Sci. 2015;370(1668):20140172. doi:10.1098/rstb.2014.0172

5. Biswal B, Zerrin Yetkin F, Haughton VM, Hyde JS. Functional connectivity in the motor cortex of resting human brain using echo-planar MRI. Magn Reson Med. 1995;34(4):537-541.

6. Honey CJ, Sporns O, Cammoun L, et al. Predicting human resting-state functional connectivity from structural connectivity. Proc Natl Acad Sci. 2009;106(6):2035-2040. doi:10.1073/pnas.0811168106
7. Greicius MD, Supekar K, Menon V, Dougherty RF. Resting-state functional connectivity reflects structural connectivity in the default mode network. Cereb Cortex. 2009;19(1):72-78. doi:10.1093/cercor/ bhn059

8. Postuma RB, Dagher A. Basal ganglia functional connectivity based on a meta-analysis of 126 positron emission tomography and functional magnetic resonance imaging publications. Cereb Cortex. 2006; 16(10):1508-1521. doi:10.1093/cercor/bhj088

9. Sherman SM, Guillery RW. Exploring the Thalamus and Its Role in Cortical Function. 2nd ed. Cambridge, Massachusetts. London, England: The MIT Press; 2006.

10. Buzsaki G, Draguhn A. Neuronal oscillations in cortical networks. Science. 2004;304(5679):1926-1929. doi:10.1126/science.1099745

11. Sherman SM. The thalamus is more than just a relay. Curr Opin Neurobiol. 2007;17(4):417-422. doi:10.1016/j.conb.2007.07.003

12. Zhang D, Snyder AZ, Shimony JS, Fox MD, Raichle ME. Noninvasive functional and structural connectivity mapping of the human thalamocortical system. Cereb Cortex. 2009;20(5):1187-1194. doi:10.1093/ cercor/bhp182

13. Fair $\mathrm{D}$, Bathula $\mathrm{D}$, Mills KL, et al. Maturing thalamocortical functional connectivity across development. Front Syst Neurosci. 2010;4:10. doi:10.3389/fnsys.2010.00009

14. Jones DT, Mateen FJ, Lucchinetti CF, Jack CJ, Welker KM. Default mode network disruption secondary to a lesion in the anterior thalamus. Arch Neurol. 2011;68(2):242-247. doi:10.1001/archneurol.2010.259

15. Oldfield RC. The assessment and analysis of handedness: the Edinburgh inventory. Neuropsychologia. 1971;9(1):97-113.

16. Yan $C$, Wang X, Zuo X, Zang Y. DPABI: data processing \& analysis for (resting-state) brain imaging. Neuroinformatics. 2016;14(3):339-351. doi:10.1007/s12021-016-9299-4

17. Li J, Gao L, Xie K, et al. Detection of functional homotopy in traumatic axonal injury. Eur Radiol. 2017;27(1):325-335. doi:10.1007/s00330$016-4302-x$

18. Ashburner J. A fast diffeomorphic image registration algorithm. Neuroimage. 2007;38(1):95-113. doi:10.1016/j.neuroimage.2007. 07.007

19. Johnston JM, Vaishnavi SN, Smyth MD, et al. Loss of resting interhemispheric functional connectivity after complete section of the corpus callosum. J Neurosci. 2008;28(25):6453-6458. doi:10.1523/ JNEUROSCI.0573-08.2008

20. Pizoli CE, Shah MN, Snyder AZ, et al. Resting-state activity in development and maintenance of normal brain function. Proc Natl Acad Sci US A. 2011;108(28):11638-11643. doi:10.1073/pnas.1109144108

21. Yu-Feng Z, Yong H, Chao-Zhe Z, et al. Altered baseline brain activity in children with ADHD revealed by resting-state functional MRI. Brain Dev. 2007;29(2):83-91. doi:10.1016/j.braindev.2006.07.002

22. Mayer AR, Mannell MV, Ling J, Gasparovic C, Yeo RA. Functional connectivity in mild traumatic brain injury. Hum Brain Mapp. 2011; 32(11):1825-1835. doi:10.1002/hbm.21151

23. Liu Y, Yu C, Liang M, et al. Whole brain functional connectivity in the early blind. Brain. 2007;130(Pt 8):2085-2096. doi:10.1093/brain/ awm 121

24. Agosta F, Valsasina P, Absinta M, et al. Sensorimotor functional connectivity changes in amyotrophic lateral sclerosis. Cereb Cortex. 2011; 21(10):2291-2298. doi:10.1093/cercor/bhr002

25. Sherman SM. Functioning of circuits connecting thalamus and cortex. Compr Physiol. 2017;7:713-739. doi:10.1002/cphy.c160032

26. Behrens TE, Johansen-Berg H, Woolrich MW, et al. Non-invasive mapping of connections between human thalamus and cortex using diffusion imaging. Nat Neurosci. 2003;6(7):750. doi:10.1038/nn1075

27. Shao Y, Wang L, Ye E, et al. Decreased thalamocortical functional connectivity after 36 hours of total sleep deprivation: evidence from resting state FMRI. PLoS One. 2013;8(10):e78830. doi:10.1371/journal.pone.0078830

28. Anticevic A, Haut K, Murray JD, et al. Association of thalamic dysconnectivity and conversion to psychosis in youth and young adults at elevated clinical risk. Am J Psychiatry. 2015;72(9):882-891. doi:10.1001/jamapsychiatry.2015.0566 
29. Alcauter S, Lin W, Smith JK, et al. Development of thalamocortical connectivity during infancy and its cognitive correlations. J Neurosci. 2014;34(27):9067-9075. doi:10.1523/JNEUROSCI.0796-14.2014

30. Woodward ND, Karbasforoushan H, Heckers S. Thalamocortical dysconnectivity in schizophrenia. Am J Psychiatry. 2012;169(10): 1092-1099. doi:10.1176/appi.ajp.2012.12010056

31. Anticevic A, Cole MW, Repovs G, et al. Characterizing thalamocortical disturbances in schizophrenia and bipolar illness. Cereb Cortex. 2013;24(12):3116-3130. doi:10.1093/cercor/bht165

32. Nair A, Treiber JM, Shukla DK, Shih P, Müller R. Impaired thalamocortical connectivity in autism spectrum disorder: a study of functional and anatomical connectivity. Brain. 2013;136(6):1942-1955. doi:10.1093/ brain/awt079

33. Raichle ME, MacLeod AM, Snyder AZ, Powers WJ, Gusnard DA, Shulman GL. A default mode of brain function. Proc Natl Acad Sci. 2001;98(2):676-682. doi:10.1073/pnas.98.2.676

34. Mitra A, Snyder AZ, Hacker CD, Raichle ME. Lag structure in restingstate fMRI. J Neurophysiol. 2014;111(11):2374-2391. doi:10.1152/ jn.00804.2013
35. Yan X, Brown AD, Lazar M, et al. Spontaneous brain activity in combat related PTSD. Neurosci Lett. 2013;547:1-5. doi:10.1016/j. neulet.2013.04.032

36. Boyer A, Deverdun J, Duffau H, et al. Longitudinal changes in cerebellar and thalamic spontaneous neuronal activity after wide-awake surgery of brain tumors: a resting-state fMRI study. Cerebellum. 2016; 15(4):451-465. doi:10.1007/s12311-015-0709-1

37. Gao L, Bai L, Zhang Y, et al. Frequency-dependent changes of local resting oscillations in sleep-deprived brain. PLoS One. 2015;10(3): e120323.

38. Zhou F, Huang S, Zhuang Y, Gao L, Gong H. Frequency-dependent changes in local intrinsic oscillations in chronic primary insomnia: a study of the amplitude of low-frequency fluctuations in the resting state. NeuroImage Clinl. 2017;15:458-465. doi:10.1016/j. nicl.2016.05.011

\section{Publish your work in this journal}

Neuropsychiatric Disease and Treatment is an international, peerreviewed journal of clinical therapeutics and pharmacology focusing on concise rapid reporting of clinical or pre-clinical studies on a range of neuropsychiatric and neurological disorders. This journal is indexed on PubMed Central, the 'PsycINFO' database and CAS, and is the official journal of The International Neuropsychiatric Association (INA). The manuscript management system is completely online and includes a very quick and fair peer-review system, which is all easy to use. Visit http://www.dovepress.com/testimonials.php to read real quotes from published authors.

Submit your manuscript here: http://www.dovepress.com/neuropsychiatric-disease-and-treatment-journal 